\title{
Vergabe der Herausgeberschaft der PVS-Sonderhefte
}

Für die Vergabe der Herausgeberschaft der PVS-Sonderhefte haben Vorstand und Beirat der DVPW auf Vorschlag der dafür eingesetzten Arbeitsgruppe das folgende Verfahren beschlossen:

1. Der Vorstand fordert interessierte Kolleginnen und Kollegen dazu auf, einen Vorschlag für die Gestaltung eines PVS-Sonderheftes zu unterbreiten. Die Vorschläge sollten auf ca. 5 Seiten die folgenden Angaben enthalten:

- Politikwissenschaftliche Relevanz des Themas

- Positionierung in der internationalen Fachdiskussion

- Innovatives Potenzial

- Zugänglichkeit für Leserinnen und Leser mit anderen wissenschaftlichen Arbeitsschwerpunkten

- Gliederung

- Autorinnen- und Autorenvorschläge

2. Auf der Grundlage der eingereichten Vorschläge trifft der Vorstand eine Entscheidung über die Herausgeberschaft und fordert die betreffenden Herausgeberinnen und Herausgeber zur Einreichung einer detaillierten Projektbeschreibung auf. Diese sollte neben einer Grobskizze des Arbeitsplanes (inhaltliche Gestaltung, zeitlicher Ablauf) ausführliche Abstracts der Beiträge enthalten. Es wird angeregt, die Beiträge über einen Call for Papers einzuwerben. Die eingereichten Skizzen werden einem anonymen Peer-review-Verfahren unterworfen. Die Gutachterinnen und Gutachter werden vom Vorstand im Benehmen mit der PVS-Redaktion bestellt. Sie unterbreiten Vorschläge zur Annahme, Ablehnung oder Überarbeitung der geplanten Beiträge. Auf der Basis des Review-Verfahrens entscheidet der Vorstand endgültig über die Herausgeberschaft und das Konzept und teilt den Herausgeberinnen und Herausgebern seine Entscheidung mit.

3. Die Herausgeberinnen und Herausgeber sind für die weitere inhaltliche Gestaltung und die zeitliche Planung des Sonderheftes verantwortlich. Es wird angeregt, in die Zeitplanung eine Autorinnen- und Autorenkonferenz aufzunehmen, bei der die Beiträge, falls dies praktikabel ist, von externen Diskutantinnen und Diskutanten besprochen werden.

Sofern Sie einen Vorschlag für die PVS-Sonderhefte 2006, 2007 oder 2008 unterbreiten möchten, senden Sie diesen bitte mit den o.g. Angaben bis zum 20. April 2004 an die Adresse der DVPW-Geschäftsstelle (DVPW, c/o. Universität Osnabrück, FB 1, 49069 Osnabrück, dvpw@uos.de) 